\section{DENTAL PRESCRIBING APP LAUNCHED}

Dental Prescribing, an app for use on iPhone, iPad and iPod touch, has been launched by the Scottish Dental Clinical Effectiveness Programme (SDCEP), part of NHS Education for Scotland (NES). Based on the popular Drug prescribing for dentistry guidance, the app will support drug prescribing within primary care dental practice by providing mobile access to the latest dental information compiled from the British National Formulary (BNF) and BNF for Children (BNFC).

\section{SELF-CONTAINED LASER}

Unlike typical portable lasers, iLase is completely self-contained in a single, integrated handpiece with no foot pedal, power cord or external controls. All the power and control you need is concentrated in the handpiece for a uniquely personal performance that can reach up to 5 watts peak power.

Its extended battery life means dentists and hygienists can experience unrestricted, focused laser productivity for longer. This is ideal when performing a comprehensive set of 25 soft-tissue and hygiene procedures including crown lengthening, gingivectomies or implant recovery. The benefits of working with iLase during these procedures also means
Dental Prescribing presents advice on conditions in an easy-to-use, problemoriented style. Drug regimens are displayed in a 'prescription-like' format to aid prescribing for both adults and children in primary care and, for the first time, are linked to the BNF website for information on drug interactions. Advice on the management of medical emergencies, including drug administration, is also provided.

The Dental Prescribing app, which has been developed in collaboration with Waracle, Dundee, is now available to purchase from the App StoreSM. the management of a range of dental no bleeding, excellent healing and no swelling, whilst curing pain and sensitivity at the same time.

For more details call your Local Digital Specialist on 08700102041 or visit www.henryschein.co.uk.

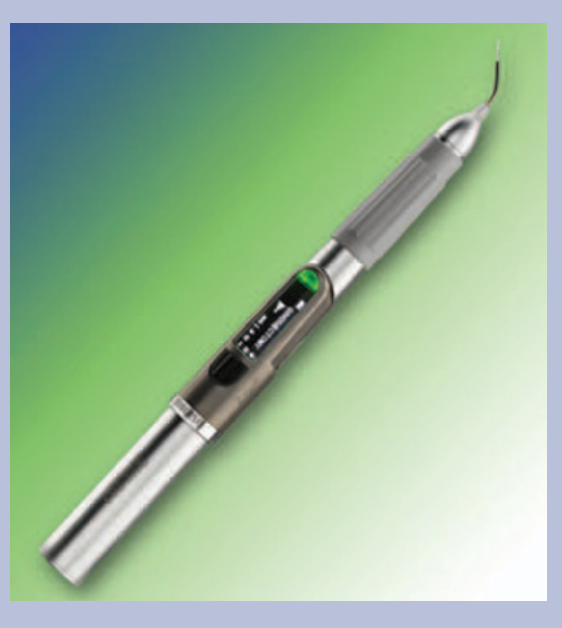

REMOVE CARIOUS WHITE SPOT LESIONS

Icon from DMG UK is an innovative caries infiltration therapy. It represents a breakthrough in microinvasive technology that reinforces and stabilises demineralised enamel without the need for drilling or sacrificing healthy tooth structure. The first product to bridge the gap between prevention (fluoride therapy) and caries restoration, Icon's micro-invasive infiltration technology can be used to treat smooth surface and proximal carious lesions up to the first third of dentine. In just one visit Icon can arrest the progression of early enamel lesions and remove carious white spot lesions.

Icon offers a simple alternative to the 'wait and see' approach, enabling dentists to offer an immediate treatment without unnecessary loss of healthy tooth structure. It prevents lesion progression and increases life expectancy for the tooth. Providing a highly aesthetic solution, carious white spot lesions treated with Icon take on the appearance of the surrounding healthy enamel. Total treatment time is about 15 minutes.

For additional information including a training video and an overview of the many international studies already being undertaken visit www.drilling-no-thanks.co.uk.

Alternatively contact your local dental dealer or DMG Dental Products (UK) Ltd on 0044 1656789401 or visit www.dmgdental.com.

of a patient's teeth using an optical scanner inside the mouth, removing the uncomfortable process of conventional impression taking. Once the scanning process is complete the files are emailed to the laboratory of choice.

Investing in the iTero system will help your procedures become more time-efficient and cost-effective.

For more information about Cadent iTero Intra-oral Scanner \& Digital Impression System call Straumann on 01293651230 or visit www. straumann.com.

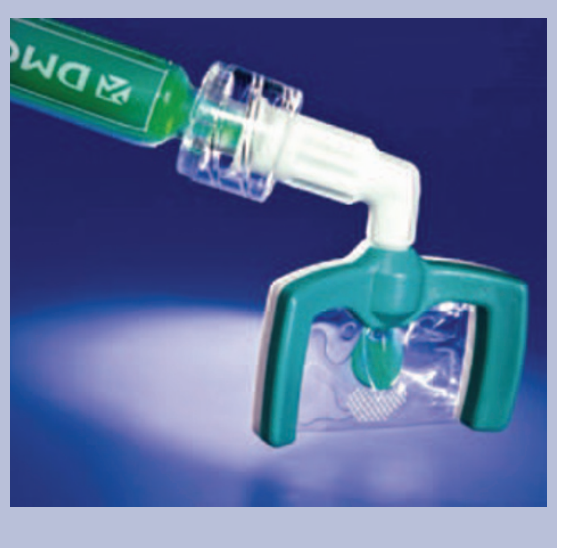

